\title{
How to Achieve Optimal Care in Early Breast Cancer with 'Less' or 'More' Treatment
}

\author{
Giuseppe Curigliano \\ Division of Early Drug Development for Innovative Therapies, Istituto Europeo di Oncologia, Milan, Italy
}

The aim of this special issue of Breast Care is to focus on areas of 'escalation' or 'de-escalation' in the adjuvant treatment of early breast cancer. That is, to identify areas where optimal care may be achieved with 'less' or 'more' treatment.

Avoidance of unnecessary or ineffective treatment should be one of the main goals in adjuvant breast oncology today. 'Do not harm' remains the main principle in medicine. To be able to follow this rule, we need to better understand the biology of breast cancer. The risk of overtreatment is real and the selection of precisely defined cohorts for clinical trials is necessary, despite the pressure of scientific ambition, pragmatism, and demands of industry. The 'add on' clinical trial design model accepts the inability to confirm that standard therapy is still necessary if a positive result from the addition of the new therapy is obtained. The same model can be applied to 'extended' adjuvant treatments in breast cancer subtypes. It is essential to escalate treatment when necessary and to de-escalate when unnecessary. Precise tools to optimally select patients and regimens for adjuvant chemotherapy in early breast cancer are missing. We need to discover biomarkers that could help identifying patients who are more likely to benefit from chemotherapy.

Research that aims at understanding the complexity of resistance to a given chemotherapy agent and the way in which resistance can be overcome is essential. Appreciation of additional diversity in biology and treatment sensitivity within the intrinsic breast cancer subtypes requires new approaches to personalize treatment as much as possible and to maximize efficacy. To achieve this goal it is fundamental to identify the functional biological targets, the cell pathway functions, the intra-tumoral biological heterogeneity, and influential host features, mostly the host stroma and immune defenses contained in the tumor microenvironment. We believe that in the near future, breast cancer treatment will be more tailored to both the individual and the tumor. There will be more investment in trials in the neoadjuvant setting in order to identify active drugs for further clinical development and to find biomarkers of response. Another potential approach for the future will be the use of gene expression arrays. Specific gene expression signatures have been proposed to predict potential benefit form chemotherapy of extended endocrine therapy [1-9].

The 'holy grail' of personalized cancer care, the major ambition of current translational research, will remain elusive unless we can find ways to identify specifically those individual patients who benefit from a given systemic therapy, avoiding the risk of overtreatment. To accomplish this we require a change in the way trials are designed to investigate a new therapy or a new therapeutic approach, but we also need to confirm that the de-escalation of interventions previously regarded as standard of care in some groups of patients can be achieved without harm. What is the level of absolute benefit that is required before accepting a new therapeutic approach as a standard of care? Escalation trials are more successful than de-escalation trials. Estimating the magnitude of clinical benefit and the perceptions of appropriate efficacy-toxicity relationships to design trials will yield more relevant results in the future treatment of early breast cancer.

\section{Disclosure Statement}

The author does not have any conflict of interest to disclose.

\section{References}

1 Harris LN, Ismaila N, McShane LM, et al.: Use of biomarkers to guide decisions on adjuvant systemic therapy for women with early-stage invasive breast cancer: American Society of Clinical Oncology clinical practice guideline. J Clin Oncol 2016;34:1134-1150.
2 Sparano JA, Gray RJ, Makower DF, et al.: Prospective validation of a 21-gene expression assay in breast cancer. N Engl J Med 2015;373:2005-2014.

3 Gluz O, Nitz UA, Christgen M, et al.: West German Study Group phase III PlanB trial: first prospective outcome data for the 21-gene recurrence score assay and concordance of prognostic markers by central and local pathology assessment. J Clin Oncol 2016;34: 2341-2349.

4 Cardoso F, van't Veer LJ, Bogaerts J, et al.: 70-gene signature as an aid to treatment decisions in early-stage breast cancer. N Engl J Med 2016;375:717-729.

\section{KARGER}

(c) 2017 S. Karger GmbH, Freiburg

Fax +497614520714
Giuseppe Curigliano, MD, PhD Division of Early Drug Development for Innovative Therapies Istituto Europeo di Oncologia Via Ripamonti 435, 20141 Milano, Italy giuseppe.curigliano@ieo.it 
5 Gnant M, Filipits M, Greil R, et al.: Predicting distant recurrence in receptor-positive breast cancer patients with limited clinicopathological risk: using the PAM50 risk of recurrence score in 1478 postmenopausal patients of the ABCSG-8 trial treated with adjuvant endocrine therapy alone. Ann Oncol 2014;25:339-345.

6 Filipits M, Rudas M, Jakesz R, et al.: A new molecula predictor of distant recurrence in ER-positive, HER2negative breast cancer adds independent information to conventional clinical risk factors. Clin Cancer Res 2011;17:6012-6020.
7 Gnant M, Sestak I, Filipits M, et al.: Identifying clinically relevant prognostic subgroups of postmenopausal women with node-positive hormone receptor-positive early-stage breast cancer treated with endocrine therapy: a combined analysis of ABCSG-8 and ATAC using the PAM50 risk of recurrence score and intrinsic subtype. Ann Oncol 2015;26:1685-1691.

8 Sgroi DC, Sestak I, Cuzick J, et al.: Prediction of late distant recurrence in patients with oestrogen-receptorpositive breast cancer: a prospective comparison of the breast-cancer index (BCI) assay, 21-gene recurrence score, and IHC4 in the TransATAC study population. Lancet Oncol 2013;14:1067-1076.

9 Dubsky P, Brase JC, Jakesz R, et al.: The EndoPredic score provides prognostic information on late distant metastases in ER+/HER2- breast cancer patients. Br J Cancer 2013;109: 2959-2964. 